\title{
Asymptomatic Candiduria among Type 1 and 2 Diabetes Mellitus Patients: Risk and Sociodemographic Factors, Prevalence, Virulence Markers and Antifungal Susceptibility
}

\author{
Olajide Joseph Akinjogunla ${ }^{1 *}$ (D) Ofon-mbuk Divine-Anthony ${ }^{1}$, Ayodele \\ Oluwaseun Ajayi ${ }^{2}$, Idongesit Udofot Etukudo ${ }^{3}$ and Idongesit Jonah Etok ${ }^{1}$ \\ ${ }^{1}$ Department of Microbiology, Faculty of Science, University of Uyo, P.M.B. 1017, Uyo, Akwa Ibom State, Nigeria. \\ ${ }^{2}$ Department of Microbiology, Faculty of Science, Federal University, Oye-Ekiti, Ekiti State, Nigeria. \\ ${ }^{3}$ Department of Microbiology, Faculty of Biological Science, Abia State University, Uturu, Abia State, Nigeria.
}

\begin{abstract}
Diabetes mellitus (DM) has been considered as one of the predisposing factors for candiduria and Candida urinary tract infections. The study determined the socio-demographic characteristics, risk factors of DM patients with asymptomatic candiduria and ascertained the prevalence, virulence factors and antifungal susceptibility of Candida isolated. Socio-demographic and risk factors were obtained via questionnaires. Microscopic, macroscopic and chemical analysis of mid-stream urine (MSU) samples were determined by microbiological method and dipsticks. The characterization, virulence factors, antibiotic susceptibility of Candida isolates were determined by conventional, mycological media and disc diffusion techniques, respectively. Of the $51 \mathrm{MSU}$ samples, $\geq 31.4 \%$ were amber and clear in colour, contained yeast cells and leukocytes; between 5.9 to $25.5 \%$ had hyaline casts, urobilinogen, epithelial cells, red blood cells, pus cells and nitrite, while the specific gravity was $\geq 1.015$. The prevalence of candiduria among subjects with respect to age, types and duration of diabetes, gender, tobacco and alcohol consumption were not significant $(p \geq 0.005)$. Candida dubliniensis and $C$. parapsilosis prevalence was highest in subjects with random blood sugar $(\mathrm{mg} / \mathrm{dL})$ of $\geq 400$ and $300-399$, respectively. Of the 39 isolates, $64.1 \%$ were Fluconazole sensitive, $10.3 \%$ were dose dependent susceptible to Ketoconazole, $\mathbf{7 4 . 4 \%}$ exhibited Voriconazole sensitivity, $100 \%$ C. dubliniensis were Clotrimazole sensitive, $\leq \mathbf{2 8 . 6 \%} \mathrm{C}$. tropicalis and C. glabrata were resistant to Amphotericin B and Itraconazole, while between $23.1 \%$ and $\mathbf{7 1 . 8 \%}$ isolates produced hydrolytic enzymes and biofilm. This study revealed the socio-demographic characteristics and risk factors among subjects and the necessity to continuously investigate pathogenic Candida against antifungal agents for effective treatments of asymptomatic candiduria in diabetes mellitus patients.
\end{abstract}

Keywords: Candiduria, Diabetes, Risk Factors, Antifungal, Susceptibility, Virulence Factors

(C) The Author(s) 2020. Open Access. This article is distributed under the terms of the Creative Commons Attribution 4.0 International License which permits unrestricted use, sharing, distribution, and reproduction in any medium, provided you give appropriate credit to the original author(s) and the source, provide a link to the Creative Commons license, and indicate if changes were made. 


\section{INTRODUCTION}

Diabetes mellitus, chronic metabolic and degenerative disorder characterized by hyperglycemia, is an increasing global health problem with numerous complications (LotfiKamran et al., 2009; Zahra et al., 2016). Human behavioural changes, lifestyle, genetics and environment factors such as high intake of refined carbohydrates, smoking and heavy consumption of alcohol have been implicated in the burgeoning prevalence of diabetes mellitus ( $\mathrm{Hu}, 2011)$. The two types of diabetes mellitus are type 1 and type 2 (Mukhtar et al., 2019). The type 1 diabetes mellitus is a heterogeneous disorder characterized by the destruction of pancreatic beta cells, resulting in absolute insulin deficiency (Maahs et al., 2010; Mukhtar et al., 2019), while type 2 consists of dysfunctions characterized by hyperglycemia and resulting from the combination of resistance to insulin action and an inadequate compensatory insulin secretory response (Mukhtar et al., 2019). Diabetes mellitus, immunosuppression, gender, age, exposure to antimicrobial agents and organ transplantation have long been considered as a predisposing factor for Candida urinary tract infections (Kauffman, 2005; Sobel et al., 2011).

The urinary tract consists of organs of the body involved in the production, storage and excretion of urine (Beer et al., 2006). The urinary tract is sterile, devoid of microorganisms but may be invaded by pathogenic organisms (Akinjogunla et al., 2010). Several studies have revealed increase in urinary tract infections (UTIs), attributable to Candida spp (Yashavanth et al., 2013). Candida species are thin-walled, dimorphic fungi ( 8 to $12 \mu \mathrm{m}$ in diameter), that reproduce by budding (Talaro and Talaro, 1996). Although, $>150$ Candida spp have been reported, only few cause human diseases (Kwon-Chung et al., 1992). Candiduria, the presence of Candida spp such as C. albicans, C. glabrata, C. krusei, C. tropicalis and $C$. parapsilosis in urine, is classified into asymptomatic and symptomatic forms. Candiduria can lead to morbidity and mortality in immunocompromised and ill-patients (Sanglard and Odds, 2002). Candida spp promptly react to environmental changes and take advantage of compromised immunity for establishment of diseases (Akinjogunla et al., 2017). The emergence of drug-resistant Candida spp is attributable to the use of prolonged and inappropriate empirical therapy which has further complicated the management of patients (Chakrabarti et al., 1996). Thus, it's important to appropriately identify the causal agents of the infection and administer proper treatment so as to prevent the disease from becoming chronic.

The virulence factors that enhance the pathogenicity of Candida spp are the: phenotypic switching (Soll, 1992); adhesins and invasins on the cell surface (Akinjogunla et al., 2019); yeast hyphal morphogenetic transformation and hydrolytic enzymes e.g. proteases, phospholipases, haemolysins and lipase (Francois et al., 2013). These hydrolytic enzymes aid in adherence, tissue penetration, invasion and destruction of host tissues (Silva et al., 2011; Akinjogunla et al., 2019). Biofilms, communities of microorganisms embedded in an extracellular matrix, similarly contribute to the pathogenicity of Candida spp and confer substantial resistance to antifungal therapy (Donlan and Costerton, 2002). The study determined the socio-demographic characteristics and risk factors of diabetic patients with asymptomatic candiduria and as well ascertained the prevalence, virulence markers / factors (amylase, lipase, phospholipase, caseinase, gelatinase, coagulase, biofilm, haemolysin) and susceptibility profiles of Candida isolated to antifungal drugs (Ketoconazole, Clotrimazole, Voriconazole, Itraconazole, Fluconazole and Amphotericin) using disc diffusion technique.

\section{MATERIALS AND METHODS Collection of Samples}

Mid-stream urine (MSU) samples were aseptically collected using sterile wide-necked, leak-proof containers from 51 diabetic patients (aged $<40$ yrs and $\geq 41 \mathrm{yrs}$ ), with random blood sugar ranging between 200 and $\geq 400 \mathrm{mg} / \mathrm{dL}$, attending some hospitals / clinics in Uyo, Akwa Ibom State. The Ethics Review Committee approval and verbal informed consent of diabetic patients were obtained prior to samples collection. The MSU samples were properly labelled and transported in a cooler box $\left(4^{\circ} \mathrm{C}\right)$ to microbiology laboratory for analysis.

\section{Administration of Questionnaires}

Structured questionnaires reflecting the socio-demographic characteristics and associated 
risk factors were administered to the participants after their verbal informed consent.

\section{Inclusion Criteria}

The diabetic patients who agreed and gave verbal consent to participate in the study.

\section{Exclusion Criteria}

The non-diabetic patients and diabetic patients who were on antibiotics within one week prior to enrolment and/or those that declined to participate in the study.

Microscopic and Macroscopic Examination of Mid-stream Urine Samples

Two loopful of each uncentrifuged MSU sample was aseptically placed on a clean grease-free slide and covered with a cover slip. The presence of epithelial cell, pus cell, yeast cell, granular cast, crystals, hyaline cast and red blood cells were observed using $10 \mathrm{x}$ and $40 \mathrm{x}$ objectives with condenser iris sufficiently closed. Macroscopic examination was done by observing colour of the MSU samples as previously described by Akinjogunla and Divine-Anthony (2013).

Chemical (Dipstick) Analysis of Mid-stream Urine Samples

The chemical analysis of MSU samples was carried out using dipsticks (Medi-Test Combi 10 SGL, Macherey-Nagel, Germany). The parameters evaluated were: urobilinogen, leukocytes, protein, ketone, bilirubin, nitrites, specific gravity and blood. The dipstick was dipped into each fresh uncentrifuged MSU sample and removed after 5-10 secs. A colour change of the dipstick within 2 mins was observed and recorded according to the manufacturer's instructions.

Mycological Analysis of Mid-stream Urine Samples

A loopful of each MSU sample was aseptically inoculated onto each plate of Sabouraud Dextrose Agar (SDA, Oxoid UK) supplemented with chloramphenicol and aerobically incubated at $35^{\circ} \mathrm{C}$ for $48 \mathrm{hrs}$. After incubation, the plates were observed for yeast growth and were subcultured onto fresh plates of SDA and incubated at $35^{\circ} \mathrm{C}$ \pm 2 for $48 \mathrm{hr}$. After incubation, the pure isolates were subcultured onto plates of CHROMagar Candida (Difco BBL., USA), aerobically incubated at $35^{\circ} \mathrm{C} \pm 2$ for $48 \mathrm{hr}$ and their colonial morphology and pigmentation on CHROMagar Candida were used for identification. The Candida species were further identified using Gram staining, temperature tolerance, chlamydospore and germ tube formation, sugar fermentation and assimilation tests. The Candida isolates were maintained on SDA slant at $4^{\circ} \mathrm{C}$.

In vitro Antifungal Susceptibility of Candida Isolates

In vitro antifungal susceptibility of Candida isolates to Ketoconazole (KET, $10 \mu \mathrm{g}$ ), Clotrimazole (CLO, $10 \mu \mathrm{g})$, Voriconazole (VOR, $1 \mu \mathrm{g})$, Itraconazole (ITR, $10 \mu \mathrm{g})$, Fluconazole (FLU, $25 \mu \mathrm{g})$, and Amphotericin B (AMP, $20 \mu \mathrm{g}$ ) was carried out using disc diffusion technique (CLSI, 2009). Ten microlitre of inoculum suspension, adjusted to turbidity of $0.5 \mathrm{McF}$ arland standards, was inoculated onto the plate of Mueller Hilton Agar supplemented with $2 \%$ glucose and $0.5 \mathrm{~g} / \mathrm{mL}$ methylene blue (GM-MHA). The GM-MHA plates were allowed to dry for 15 mins before antifungal discs were aseptically placed on the surfaces of the culture plates using sterile forceps. The plates were aerobically incubated at $35^{\circ} \mathrm{C} \pm 2$ for $24 \mathrm{hr}$, inhibition zones after incubation were observed, measured in millimeters $(\mathrm{mm})$ using a ruler and interpreted as sensitive (S), dose dependent susceptible (DDS) and resistant (R) was made as follows: ITR, AMP and VOR (S: $\geq 16$, DDS: 10-15, R $\leq$ 9), FLU (S: $\geq 19$, DDS: $15-18, R \leq 14)$, KET (S: $\geq 30$, DDS: $23-29, R \leq 22)$ and CLO (S: $\geq 20$, DDS: 12-19, $\mathrm{R} \leq 11$ ).

Detection of Phospholipase, Lipase, Gelatinase, Caseinase and Hemolysin Producing Candida isolates

Phospholipase, lipase, gelatinase, caseinase and hemolysin producing Candida isolates were detected using egg yolk agar (SDA, $2 \%$ egg yolk), tributyrin agar (SDA, 1\% tributyrin), gelatin agar (SDA, $1 \%$ gelatin), skim milk agar (SDA, 1\% skim milk) and human blood - Sabouraud Dextrose Agar (SDA, 3 \% glucose, 5\% human blood) respectively (Deorukhkar et al., 2014; Akinjogunla et al., 2107). Ten microliters of Candida isolate suspension, adjusted to turbidity of $0.5 \mathrm{McF}$ arland standards, was inoculated onto each plate of egg yolk agar, tributyrin agar, gelatin agar, skim milk agar and human blood-SDA. All the plates were aerobically incubated at $35^{\circ} \mathrm{C} \pm 2$ for $72 \mathrm{hrs}$. After incubation, transparent zones around the colonies were observed and considered positive for production of phospholipase, lipase, gelatinase, caseinase and hemolysin, respectively. 
Detection of Amylase producing Candida isolates Amylase producing Candida isolates was detected using starch agar (SDA, 2\% starch). The Candida isolates were streaked onto starch agar plates and aerobically incubated for $48-72 \mathrm{hr}$ at $35^{\circ} \mathrm{C} \pm 2$. After incubation, 3 drops of $10 \%$ Lugol iodine was put on the culture plates and allowed to react for $10 \mathrm{~min}$. Clear zone around the colony indicated the production of amylase (Akinjogunla et al., 2107).

Detection of Biofilm Producing Candida Isolates

A loopful of each Candida isolate from overnight agar plate was aseptically inoculated onto each tube of Glucose-Sabouraud Dextrose Broth (10 $\mathrm{ml} \mathrm{SDB}, 2 \%$ glucose). Each tube was incubated aerobically for $48 \mathrm{hrs}$ at $35^{\circ} \mathrm{C} \pm 2$. After incubation, the contents of each tube were poured out, each tube washed with phosphate buffer saline (PBS, pH 7.2), dried and stained with safranin (1\%). Excess stain in each tube was rinsed with PBS and tubes dried in an inverted position. A biofilm formation was indicated when a visible film lined the wall and bottom of the tube (Deorukhkar et al., 2014; Akinjogunla et al., 2107).

Detection of Coagulase Producing Candida Isolates

The production of coagulase by Candida isolates was determined using EDTA-human plasma. Ten microlitre of each Candida isolate from overnight agar plate was aseptically inoculated onto each tube of EDTA-human plasma. Each tube was incubated at $35^{\circ} \mathrm{C} \pm 2$ and observed for clot formation after 2, 4, 6 and $24 \mathrm{hr}$. The presence of clot that could not be resuspended by gentle shaking indicated positive for coagulase production (Rodrigues et al., 2003).

\section{Statistical Analysis}

The Statistical Package for Social Sciences (IBM SPSS Version 22.0. Armonk, NY: IBM Corp.) was used for data analysis. The significant difference in the socio-demographic characteristics and associated risk factors among the diabetic patients at $p \leq 0.05$ were determined using chisquare $(\chi 2)$ test. Descriptive data were presented as charts and percentages.

\section{RESULTS}

The microscopic analysis of MSU samples based on age and gender of subjects are shown on Table 1. Of the 51 samples collected, $13.7 \%$, $25.5 \%, 35.3 \%, 9.8 \%, 13.7 \%, 5.9 \%$ and $11.8 \%$ had epithelial cells, pus cells, yeast cells, granular cast, crystals, hyaline casts and red blood cells (RBCs), respectively. The occurrence of epithelial cells, pus cells, yeast cells, crystals, hyaline casts and RBCs was higher in female subjects and those aged $\geq 40$ yrs than male subjects and those aged $<40$ yrs. Only $5.9 \%$ MSU samples of subjects aged $\geq 40$ yrs contained hyaline casts, while none of the samples of subjects within age group $<40$ yrs had hyaline casts (Table 1 ).

The results of macroscopic and dipstick analysis of MSU samples are shown in Table 2. The colour of MSU samples was: pale yellow and clear $11(21.6 \%)$, pale yellow and turbid 6(11.8\%), yellow and turbid $3(5.9 \%)$, colourless and clear 7(13.7\%); amber and clear 10 (35.3\%); amber and turbid $6(11.8 \%)$. Of the $51 \mathrm{MSU}$ samples collected,

Table 1. Microscopic Analysis of Urine Samples of Diabetic Patients Based on Age and Gender

\begin{tabular}{|c|c|c|c|c|c|c|c|c|c|}
\hline \multirow[b]{2}{*}{ Parameters } & & \multirow{2}{*}{$\begin{array}{l}\text { No of } \\
\text { Samples } \\
\text { Collected }\end{array}$} & \multicolumn{7}{|c|}{ No (\%) of Occurrence } \\
\hline & & & $\begin{array}{l}\text { Epithelial } \\
\text { Cell }\end{array}$ & $\begin{array}{l}\text { Pus } \\
\text { Cell }\end{array}$ & $\begin{array}{l}\text { Yeast } \\
\text { Cell }\end{array}$ & $\begin{array}{l}\text { Granular } \\
\text { Cast }\end{array}$ & Crystals & $\begin{array}{l}\text { Hyaline } \\
\text { Cast }\end{array}$ & $\mathrm{RBC}$ \\
\hline \multirow[t]{3}{*}{ Age (yrs) } & $<40$ & 13 & $1(7.7)$ & $3(23.1)$ & $4(30.8)$ & $2(15.4)$ & $1(7.7)$ & $0(0.0)$ & $1(7.7)$ \\
\hline & $\geq 40$ & 38 & $6(15.8)$ & $10(26.3)$ & $14(36.8)$ & $3(7.9)$ & $6(15.8)$ & $3(7.9)$ & $5(13.2)$ \\
\hline & Total & 51 & 7 (13.7) & $13(25.5)$ & $18(35.3)$ & $5(9.8)$ & 7 (13.7) & $3(5.9)$ & $6(11.8)$ \\
\hline \multicolumn{10}{|l|}{ Gender } \\
\hline & Male & 29 & $3(10.3)$ & $5(17.2)$ & $8(27.6)$ & $4(13.8)$ & $3(10.3)$ & $1(3.4)$ & $1(3.4)$ \\
\hline & Female & 22 & $4(18.2)$ & $8(36.4)$ & $10(45.5)$ & $1(4.5)$ & $4(18.2)$ & $2(9.1)$ & $5(22.7)$ \\
\hline & Total & 51 & 7 (13.7) & $13(25.5)$ & $18(35.3)$ & $5(9.8)$ & $7(13.7)$ & $3(5.9)$ & $6(11.8)$ \\
\hline
\end{tabular}

RBC: Red Blood Cell. values in parentheses expressed the percentages. 
Table 2. Macroscopic and Chemical Analysis of Urine Samples of Diabetic Patients $(n=51)$

\begin{tabular}{|c|c|c|c|c|}
\hline \multirow[b]{2}{*}{ Variables } & \multirow[b]{2}{*}{ Categories } & \multicolumn{3}{|c|}{ No (\%) of Occurrence } \\
\hline & & $\begin{array}{l}\text { Male } \\
\text { (29) }\end{array}$ & $\begin{array}{l}\text { Female } \\
(22)\end{array}$ & $\begin{array}{l}\text { Total } \\
\text { No (\%) }\end{array}$ \\
\hline \multirow[t]{6}{*}{ Appearance } & PYC & $7(24.1)$ & $4(18.2)$ & $11(21.6)$ \\
\hline & PYT & $3(10.3)$ & $3(13.6)$ & $6(11.8)$ \\
\hline & $\mathrm{YT}$ & $2(6.9)$ & $1(4.5)$ & $3(5.9)$ \\
\hline & $\mathrm{CC}$ & $4(13.8)$ & $3(13.6)$ & $7(13.7)$ \\
\hline & $A C$ & 11 (37.9) & $7(31.8)$ & $18(35.3)$ \\
\hline & AT & $2(6.9)$ & $4(18.2)$ & $6(11.8)$ \\
\hline \multirow[t]{3}{*}{ Protein } & ++ & $2(6.9)$ & $1(4.5)$ & $3(5.9)$ \\
\hline & + & $4(13.8)$ & $3(13.6)$ & $7(13.7)$ \\
\hline & - & $23(79.3)$ & 18 (81.8) & $41(80.4)$ \\
\hline \multirow[t]{3}{*}{ Ketone } & ++ & $5(17.2)$ & $3(13.6)$ & $8(15.7)$ \\
\hline & + & $4(13.8)$ & $4(18.2)$ & $8(15.7)$ \\
\hline & - & $20(69.0)$ & $15(68.2)$ & $35(68.6)$ \\
\hline \multirow[t]{3}{*}{ Bilirubin } & ++ & $0(0.0)$ & $0(0.0)$ & $0(0.0)$ \\
\hline & + & $1(3.4)$ & $3(13.6)$ & $4(7.8)$ \\
\hline & - & $28(96.6)$ & $19(86.4)$ & $47(92.2)$ \\
\hline \multirow[t]{2}{*}{ Nitrite } & + & $5(17.2)$ & $1(4.5)$ & $6(11.8)$ \\
\hline & - & $24(82.8)$ & 21 (95.5) & $45(88.2)$ \\
\hline \multirow[t]{3}{*}{ Urobilinogen } & ++ & $1(3.4)$ & $0(0.0)$ & $1(2.0)$ \\
\hline & + & $1(3.4)$ & $1(4.5)$ & $2(4.0)$ \\
\hline & - & 27 (93.1) & 21 (95.5) & 48 (94.1) \\
\hline \multirow[t]{3}{*}{ Leukocytes } & ++ & $3(10.3)$ & $3(13.6)$ & 6 (11.8) \\
\hline & + & $5(17.2)$ & $7(31.8)$ & $12(23.5)$ \\
\hline & - & $21(72.4)$ & $12(54.5)$ & 33 (64.7) \\
\hline \multirow[t]{3}{*}{ Blood } & ++ & $0(0.0)$ & $1(4.5)$ & $1(2.0)$ \\
\hline & + & $1(3.4)$ & $4(18.2)$ & $5(9.8)$ \\
\hline & - & $28(96.6)$ & $17(77.3)$ & 45 (88.2) \\
\hline Specific & 1.000 & $2(6.9)$ & $1(4.5)$ & $3(5.9)$ \\
\hline \multirow[t]{3}{*}{ Gravity } & 1.005 & $8(27.6)$ & $6(27.3)$ & $14(27.5)$ \\
\hline & 1.010 & $4(13.8)$ & $3(13.6)$ & $7(13.7)$ \\
\hline & 1.015 & 15 (51.7) & $12(54.5)$ & 27 (52.9) \\
\hline
\end{tabular}

-: Negative; +: Present in low amount; ++: Present in high amount; PYC: Pale yellow and clear; PYT: Pale yellow and turbid; YT: Yellow and turbid; CC: Colourless and clear; AC: Amber and clear; AC: Amber and turbid

6 samples had leukocytes (+), 12 samples had leukocytes (++); $19.6 \%$ were positive for proteins; $15.7 \%$ contained ketone $(+), 15.7 \%$ had ketone $(++), 4.0 \%$ contained urobilinogen $(+), 2.0 \%$ had urobilinogen $(++), 11.8 \%$ contained blood; $5.9 \%$ had specific gravity (SG) of $1.000 ; 27.5 \%$ had SG of $1.005 ; 13.7 \%$ had SG of 1.010 , SG of $52.9 \%$ was 1.015 , while nitrites and bilirubin were present in $11.8 \%$ and $7.8 \%$, respectively.

The socio-demographic characteristics and risk factors of subjects are presented in Table 3. Of the $51 \mathrm{MSU}$ samples from subjects, $35.3 \%$ had Candida isolates growth, indicating candiduria, while $64.7 \%$ had no growth of Candida isolates. Of 18 subjects with candiduria, 9(50.0\%) were males and 9(50.0\%) were females, giving a gender ratio of $1: 1$. The hypertensive subjects had higher prevalence of candiduria $(8 / 20 ; 40.0 \%)$ than non-hypertensive subjects $(10 / 31 ; 32.3 \%)$. The prevalence of candiduria was lower among subjects with type I diabetes, family history of diabetes and duration of diabetes $<5$ yrs than among subjects with type 2 diabetes, duration of diabetes $\geq 5$ yrs and without family history of diabetes. There was no statistically significant relationship between the prevalence of candiduria 
Table 3. Socio-Demographic Characteristics and Risk Factors of Diabetic Patients

\begin{tabular}{|c|c|c|c|c|c|}
\hline \multirow[b]{2}{*}{$\begin{array}{l}\text { Socio-Demographic } \\
\text { Characteristics /Risk } \\
\text { Factors }\end{array}$} & \multirow[b]{2}{*}{$\begin{array}{l}\text { No of Samples } \\
\text { Collected }\end{array}$} & \multicolumn{2}{|c|}{ No (\%) of Subjects } & \multirow[b]{2}{*}{$\chi^{2}$} & \multirow[b]{2}{*}{$p$-value } \\
\hline & & $\begin{array}{l}\text { with } \\
\text { Candiduria }\end{array}$ & $\begin{array}{l}\text { without } \\
\text { Candiduria }\end{array}$ & & \\
\hline \multicolumn{6}{|l|}{ Age (yrs) } \\
\hline$<40$ & 13 & $3(23.1)$ & 10 (76.9) & 1.14 & 0.286 \\
\hline$\geq 40$ & 38 & $15(39.5)$ & $23(60.5)$ & & \\
\hline \multicolumn{6}{|l|}{ Gender } \\
\hline Male & 29 & $9(31.0)$ & $20(69.0)$ & 0.534 & 0.465 \\
\hline Female & 22 & $9(40.9)$ & $13(59.1)$ & & \\
\hline \multicolumn{6}{|l|}{ Marital Status } \\
\hline Single & 7 & $2(28.6)$ & $5(71.4)$ & & \\
\hline Married & 30 & $11(36.7)$ & $19(63.3)$ & 0.164 & 0.921 \\
\hline \multicolumn{6}{|l|}{ Occupation } \\
\hline Teaching & 8 & $2(25.0)$ & $6(75.0)$ & & \\
\hline Civil / Public Service & 21 & $7(33.3)$ & $14(66.7)$ & 0.717 & 0.869 \\
\hline Self -Employed & 12 & $5(41.7)$ & $7(58.3)$ & & \\
\hline \multicolumn{6}{|l|}{ Educational Status } \\
\hline Primary & 10 & $4(40.0)$ & $6(60.0)$ & & \\
\hline Secondary & 9 & $3(33.3)$ & $6(66.7)$ & 1.45 & 0.693 \\
\hline Tertiary & 19 & $5(26.3)$ & $14(73.7)$ & & \\
\hline None & 13 & $6(46.2)$ & $7(53.8)$ & & \\
\hline \multicolumn{6}{|l|}{ Type of Diabetes } \\
\hline Type 1 & 17 & $5(29.4)$ & $12(70.6)$ & 0.386 & 0.534 \\
\hline Type 2 & 34 & $13(38.2)$ & 21 (61.8) & & \\
\hline \multicolumn{6}{|l|}{ Duration of Diabetes } \\
\hline$<5$ yrs & 23 & $7(30.4)$ & 16 (69.6) & 0.433 & 0.510 \\
\hline$\geq 5$ yrs & 28 & $11(39.3)$ & $17(60.7)$ & & \\
\hline \multicolumn{6}{|l|}{ Family History of DM } \\
\hline Yes & 11 & $3(27.3)$ & $8(72.7)$ & 0.395 & 0.530 \\
\hline No & 40 & $15(37.5)$ & $25(62.5)$ & & \\
\hline \multicolumn{6}{|l|}{ Alcohol Consumption } \\
\hline Yes & 17 & $7(41.2)$ & $10(58.8)$ & 0.386 & 0.534 \\
\hline No & 34 & $11(32.4)$ & $23(67.6)$ & & \\
\hline \multicolumn{6}{|l|}{ Tobacco Intake } \\
\hline Yes & 7 & $3(42.9)$ & $4(57.1)$ & 0.203 & 0.652 \\
\hline No & 44 & $15(34.1)$ & $29(65.9)$ & & \\
\hline \multicolumn{6}{|l|}{ Blood Pressure } \\
\hline Hypertensive & 20 & $8(40.0)$ & $12(60.0)$ & 0.319 & 0.572 \\
\hline Non-Hypertensive & 31 & $10(32.3)$ & $21(67.7)$ & & \\
\hline
\end{tabular}

DM: Diabetes Mellitus

among subjects with respect to age $\left(\chi^{2}=1.14\right.$ $p=0.286)$, marital status $(p=0.921)$, occupations ( $p=0.869)$, educational status $(p=0.693)$, type of diabetes $(p=0.534)$, duration of diabetes $(p=$ $0.510)$, family history of diabetes $(p=0.530)$ and alcohol consumption ( $p=0.534)$. The prevalence of candiduria was higher among subjects that smoked tobacco (42.9\%) than non-tobacco smokers (34.1\%), but there was no statistically significant difference $\left(\chi^{2}=0.203, p=0.652\right)$ (Table 3). 
The percentage occurrence of Candida isolates in MSU samples of subjects in deceasing order was: C. albicans $(33.3 \%)>$ C. glabrata $(23.1 \%)>$ C. tropicalis $(17.9 \%)>$ C. krusei $(12.8 \%)$ $>$ C. parapsilosis $(7.7 \%)>$ C. dubliniensis $(5.1 \%)$ (Fig 1). With regards to total Candida isolates $(n=39)$ obtained, subjects aged $\geq 40$ yrs had $79.5 \%$ isolates; subjects aged $<40$ yrs had $20.5 \%$ isolates; male subjects (46.2\%) and female subjects (53.8\%) isolates. The predominant candida isolate in female and male subjects was $C$. albicans and $C$. parapsilosis, respectively (Table 4). C. dubliniensis prevalence was found to be highest $(100 \%)$ in subjects with random blood sugar (RBS) of $\geq 400$ $\mathrm{mg} / \mathrm{dL}$, while C. krusei and C. parapsilosis was predominant candida isolate in subjects with RBS (mg/dL) of 200-299 and 300-399, respectively (Table 4).

The results showed that $64.1 \%$ Candida isolates were Fluconazole (FLU) sensitive, while $24.5 \%$ were FLU resistant (Table 5). Of the 39 Candida isolates, $71.8 \%, 10.3 \%$ and $17.9 \%$ were sensitive, dose dependent susceptible (DDS) and resistant to Ketoconazole (KET) respectively; $74.4 \%$ isolates were sensitive to Voriconazole (VOR), $12.8 \%$ were DDS, while $12.8 \%$ isolates were resistant. All (100\%) C. dubliniensis were sensitive to Clotrimazole (CLO), KET and FLU; between $22.2 \%$ and $28.6 \%$ C. tropicalis and C. glabrata were resistant to Amphotericin B and Itraconazole (ITR);

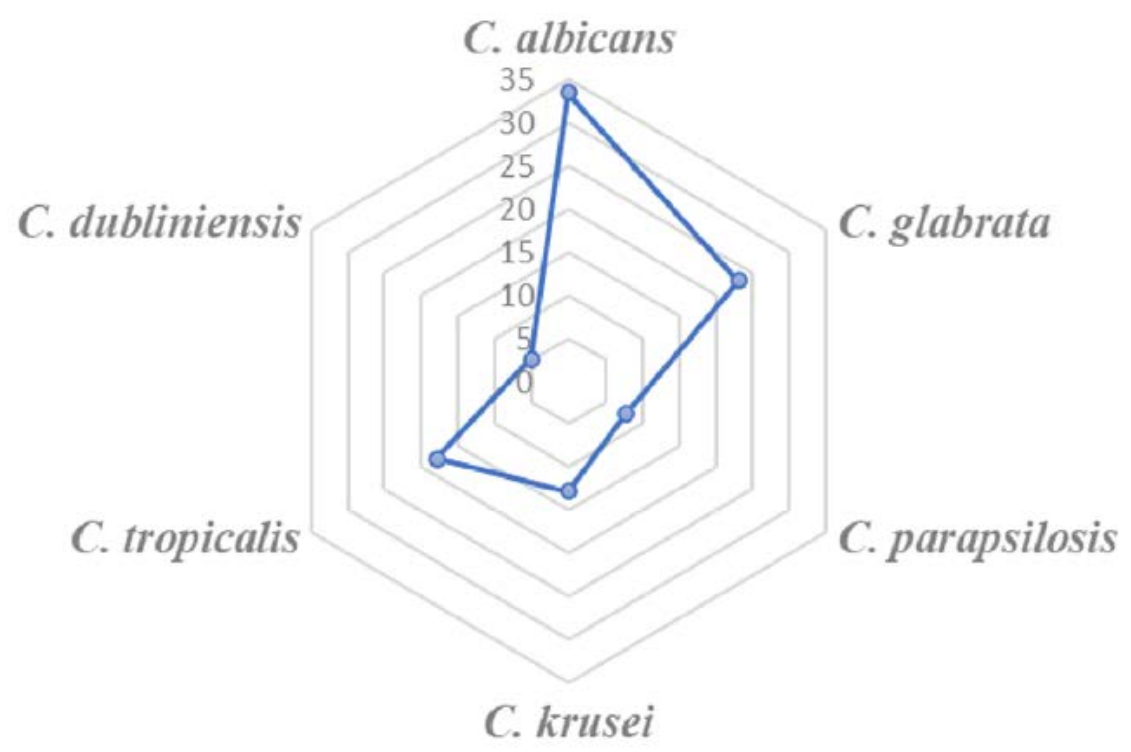

Fig. 1. Percentage Occurrences of Candida Isolates in Urine Samples of Diabetic Patients

Table 4. Distribution of Candida Isolates Based on Age, Gender and RBS Values of Diabetic (Candiduric) Patients

\begin{tabular}{|c|c|c|c|c|c|c|c|c|}
\hline \multirow[t]{2}{*}{ Candida Isolates } & \multirow[t]{2}{*}{$\begin{array}{l}\text { No of } \\
\text { Isolates }\end{array}$} & \multicolumn{2}{|c|}{$\begin{array}{l}\text { No (\%) of Occurence } \\
\text { Age (yrs) }\end{array}$} & \multicolumn{2}{|c|}{ Gender } & \multicolumn{3}{|c|}{ Random Blood Sugar (mg/dL) } \\
\hline & & $<40$ & $\geq 40$ & Male & Female & 200-299 & $300-399$ & $\geq 400$ \\
\hline C. albicans & 13 & $3(23.1)$ & $10(76.9)$ & $4(30.8)$ & $9(69.2)$ & $3(23.1)$ & $4(30.8)$ & $6(46.2)$ \\
\hline C. glabrata & 9 & $2(22.2)$ & $7(77.8)$ & $5(55.6)$ & $4(44.4)$ & $2(22.2)$ & $5(55.6)$ & $2(22.2)$ \\
\hline C. krusei & 3 & $0(0.0)$ & $3(100)$ & $1(33.3)$ & $2(66.7)$ & $3(100)$ & $0(0.0)$ & $0(0.0)$ \\
\hline C. parapsilosis & 5 & $2(40.0)$ & $3(60.0)$ & $3(60.0)$ & $2(40.0)$ & $0(0.0)$ & $4(80.0)$ & $1(20.0)$ \\
\hline C. tropicalis & 7 & $1(14.3)$ & $6(85.7)$ & $4(57.1)$ & $3(42.9)$ & $1(14.3)$ & $2(28.6)$ & $4(57.1)$ \\
\hline C. dubliniensis & 2 & $0(0.0)$ & $2(100)$ & $1(50.0)$ & $1(50.0)$ & $0(0.0)$ & $0(0.0)$ & $2(100)$ \\
\hline Total & 39 & $8(20.5)$ & $31(79.5)$ & $18(46.2)$ & $21(53.8)$ & $9(23.1)$ & 15 (38.5) & $15(38.5)$ \\
\hline
\end{tabular}

RBS: Random Blood Sugar; Values in parentheses expressed the percentage of Candida isolates 
Table 5. Antifungal Susceptibility of Candida Isolates from Diabetic (Candiduric) Patients $(\mathrm{n}=39)$

\begin{tabular}{|c|c|c|c|c|c|c|c|c|}
\hline \multirow{2}{*}{$\begin{array}{l}\text { Antifungal } \\
\text { Drug }\end{array}$} & & \multicolumn{6}{|c|}{ Candida Isolates } & \multirow[b]{2}{*}{$\begin{array}{l}\text { Total } \\
\text { No(\%) }\end{array}$} \\
\hline & & $\begin{array}{l}\text { C. albicans } \\
\text { No(\%) }\end{array}$ & $\begin{array}{l}\text { C. glabrata } \\
\text { No(\%) }\end{array}$ & $\begin{array}{l}\text { C. krusei } \\
\text { No(\%) }\end{array}$ & $\begin{array}{l}\text { C. parapsilosis } \\
\mathrm{No}(\%)\end{array}$ & $\begin{array}{l}\text { C. tropicalis } \\
\mathrm{No}(\%)\end{array}$ & $\begin{array}{l}\text { C. dubliniensis } \\
\text { No(\%) }\end{array}$ & \\
\hline & $S$ & $7(53.8)$ & $6(66.7)$ & $2(66.7)$ & $4(80.0)$ & $4(57.1)$ & $2(100)$ & $25(64.1)$ \\
\hline \multirow[t]{3}{*}{ Fluconazole } & DDS & $3(23.1)$ & $1(11.1)$ & $0(0.0)$ & $1(20.0)$ & $1(14.3)$ & $0(0.0)$ & $6(15.4)$ \\
\hline & $\mathrm{R}$ & $3(23.1)$ & $2(22.2)$ & $1(33.3)$ & $0(0.0)$ & $2(28.6)$ & $0(0.0)$ & $8(20.5)$ \\
\hline & $S$ & 10 (76.9) & $6(66.7)$ & $3(100)$ & $3(60.0)$ & $5(71.4)$ & $1(50.0)$ & $28(71.8)$ \\
\hline \multirow[t]{3}{*}{ Ketoconazole } & DDS & $0(0.0)$ & $2(22.2)$ & $0(0.0)$ & $1(20.0)$ & $0(0.0)$ & $1(50.0)$ & $4(10.3)$ \\
\hline & $\mathrm{R}$ & $3(23.1)$ & $1(11.1)$ & $0(0.0)$ & $1(20.0)$ & $2(28.6)$ & $0(0.0)$ & 7 (17.9) \\
\hline & $\mathrm{S}$ & $9(69.2)$ & $8(88.9)$ & $2(66.7)$ & $3(60.0)$ & $5(71.4)$ & $2(100)$ & $29(74.4)$ \\
\hline \multirow[t]{3}{*}{ Voriconazole } & DDS & $1(7.7)$ & $0(0.0)$ & $1(33.3)$ & $2(40.0)$ & $1(14.3)$ & $0(0.0)$ & $5(12.8)$ \\
\hline & $\mathrm{R}$ & $3(23.1)$ & $1(11.1)$ & $0(0.0)$ & $0(0.0)$ & $1(14.3)$ & $0(0.0)$ & $5(12.8)$ \\
\hline & $\mathrm{S}$ & 10 (76.9) & $6(66.7)$ & $2(66.7)$ & $4(80.0)$ & $5(71.4)$ & $1(50.0)$ & $28(71.8)$ \\
\hline \multirow[t]{3}{*}{ Amphotericin B } & DDS & $0(0.0)$ & $1(11.1)$ & $0(0.0)$ & $1(20.0)$ & $0(0.0)$ & $1(50.0)$ & $3(7.7)$ \\
\hline & $\mathrm{R}$ & $3(23.1)$ & $2(22.2)$ & $1(33.3)$ & $0(0.0)$ & $2(28.6)$ & $0(0.0)$ & $8(20.5)$ \\
\hline & $\mathrm{S}$ & $11(84.6)$ & $5(55.6)$ & $2(66.7)$ & $3(60.0)$ & $4(57.1)$ & $0(0.0)$ & $25(64.1)$ \\
\hline \multirow[t]{2}{*}{ Itraconazole } & DDS & $1(7.7)$ & $2(22.2)$ & $1(33.3)$ & $0(0.0)$ & $1(14.3)$ & $1(50.0)$ & $6(15.4)$ \\
\hline & $\mathrm{R}$ & $1(7.7)$ & $2(22.2)$ & $0(0.0)$ & $2(40.0)$ & $2(28.6)$ & $1(50.0)$ & $8(20.5)$ \\
\hline \multirow{3}{*}{ Clotrimazole } & $S$ & $9(69.2)$ & 8 (88.9) & $3(100)$ & $3(60.0)$ & $5(71.4)$ & $2(100)$ & $30(76.9)$ \\
\hline & DDS & $1(7.7)$ & $0(0.0)$ & $0(0.0)$ & $1(20.0)$ & $1(14.3)$ & $0(0.0)$ & $3(7.7)$ \\
\hline & $\mathrm{R}$ & $3(23.1)$ & $1(11.1)$ & $0(0.0)$ & $1(20.0)$ & 1 (14.3) & $0(0.0)$ & $6(15.4)$ \\
\hline
\end{tabular}

S: Sensitive; DDS: Dose Dependent Susceptible; R: Resistant; Values in parentheses expressed the percentage of Candida isolates.

Table 6. Virulence Factors of Candida Isolates from Diabetic (Candiduric) Patients

\begin{tabular}{llllllllll}
\hline & \multirow{2}{*}{$\begin{array}{l}\text { Nondida } \\
\text { Isolates }\end{array}$} & Isolates & \multicolumn{7}{c}{ No (\%) of Occurence } \\
\cline { 3 - 10 } & & AMY & LIP & PHO & CAS & GEL & COA & HAE & BIO \\
\hline C. albicans & 13 & $7(53.8)$ & $5(38.5)$ & $8(61.5)$ & $10(76.9)$ & $9(69.2)$ & $6(46.2)$ & $11(84.6)$ & $5(38.5)$ \\
C. glabrata & 9 & $4(44.4)$ & $3(33.3)$ & $5(55.6)$ & $6(66.7)$ & $5(55.6)$ & $2(22.2)$ & $5(55.6)$ & $2(22.2)$ \\
C. krusei & 3 & $0(0.0)$ & $1(33.3)$ & $2(66.7)$ & $2(66.7)$ & $3(100)$ & $0(0.0)$ & $2(66.7)$ & $0(0.0)$ \\
C. parapsilosis & 5 & $1(20.0)$ & $0(0.0)$ & $2(40.0)$ & $2(40.0)$ & $1(20.0)$ & $1(20.0)$ & $3(60.0)$ & $1(20.0)$ \\
C. tropicalis & 7 & $3(42.9)$ & $2(28.6)$ & $4(57.1)$ & $4(57.1)$ & $3(42.9)$ & $1(14.3)$ & $5(71.4)$ & $1(14.3)$ \\
C. dubliniensis & 2 & $0(0.0)$ & $1(50.0)$ & $2(100)$ & $1(50.0)$ & $2(100)$ & $0(0.0)$ & $2(100)$ & $0(0.0)$ \\
Total & 39 & $15(38.5)$ & $12(30.8)$ & $23(59.0)$ & $25(64.1)$ & $24(61.5)$ & $10(25.6)$ & $28(71.8)$ & $9(23.1)$
\end{tabular}

AMY: Amylase; LIP: Lipase; PHO: Phospholipase; CAS: Caseinase; GEL: Gelatinase; COA: Coagulase; BIO: Biofilm; HAE: Haemolysin; Values in parentheses expressed the percentage occurrences of Candida isolates.

$\leq 23.1 \%$ C. parapsilosis and C. albicans were CLO resistant, while $33.3 \%$ C. krusei were DDS to VOR and ITR (Table 5).

Table 6 shows the distribution of virulence factors in Candida isolates from MSU samples of subjects. The percentage occurrence of each virulence factor was as follows: $38.5 \%$ amylase, $30.8 \%$ lipase, $25.6 \%$ coagulase and $23.1 \%$ biofilm. A higher prevalence, > 50\%, was observed for haemolysin, phospholipase, gelatinase and caseinase (71.8\%, 59.0\%, 61.5\% and $64.1 \%$, respectively). Among candida isolates showing biofilm formation were $C$. albicans $(n=5)$, C. glabrata $(n=2), C$. parapsilosis $(n=1)$ and $C$. tropicalis $(n=1)$ while none of the $C$. dubliniensis and $C$. krusei were amylase, coagulase and biofilm producers (Table 6). 


\section{DISCUSSION}

Candida infections have increased vastly among immuno-compromised patients especially those with diabetic mellitus (DM). In this study, microscopic examinations of MSU of DM patients revealed the presence of epithelial cells, pus cells, yeast cells, crystals, hyaline casts and RBCs. The $13.7 \%$ MSU with epithelial cells and crystals obtained in this study was $\leq 27.5 \%$ epithelial cells and crystals obtained by Abebe et al. (2019) among DM patients at University of Gondar Hospital, Ethiopia. The presence of pus cells and RBCs in MSU had been reported by Akinjogunla and Divine-Anthony (2013) and Matulewicz and Meeks (2016), respectively. The presence of RBCs in urine may be attributed to lower UTI (cystitis), upper UTI (pyelonephritis) and cancer of the urinary tract, which are directly or indirectly related to DM (Matulewicz and Meeks, 2016). In our study, $25.5 \%$ DM patients had pus cells in their MSU and this value was $<35.5 \%$ reported by Abdulla et al. (2015). The pus cells are white blood cells that have succumbed in defense of the body against pathogens that invade it, therefore their presence indicates microbial infections. The MSU of DM patients in this study had specific gravity of $\leq 1.015$ and also contained leukocytes, proteins, ketone, urobilinogen, blood, nitrites and bilirubin. The percentage of MSU with protein (19.6\%), ketone (31.4\%) and leukocytes (35.3\%) in our study was higher than $11.3 \%, 4.5 \%$ and $24.9 \%$ for protein, ketone and leukocytes, respectively obtained by Abebe et al. (2019) among DM patients at University of Gondar Hospital, Ethiopia. The presence of nitrite has a predictive value for UTIs (Akinjogunla and Divine-Anthony, 2013) and the value obtained for nitrite (11.8\%) in this study was lower than $21.5 \% \mathrm{MSU}$ with nitrite reported by Fernandes et al. (2018).

The prevalence of candiduria was more in subjects aged $\geq 40$ than in subjects $<40 \mathrm{yrs}$ and this substantiated the earlier report of Hassaneen et al. (2014) and Abebe et al. (2019) that candiduria was more frequent in elderly patients. Several reports have showed frequency of candiduria to be more in diabetic women than in diabetic men (Janifer et al., 2009; Abdulla et al., 2015) and this study similarly confirmed these reports. The hypertensive subjects had higher prevalence of candiduria than non-hypertensive subjects in our study and this was in conformity with a study conducted by Muller et al. (2007) in which diabetic patients were more at risk for candiduria / UTI than hypertensive patients without diabetes. Other predisposing factors such as duration of diabetes, family history of diabetes, alcohol in-take and tobacco consumption were observed to be associated with occurrence of candiduria in this study. The prevalence of candiduria was more in subjects with duration of diabetes $\geq 5$ yrs than those subjects with duration of diabetes $<5$ yrs and this was similar to the findings of Janifer et al. (2009).

Candida albicans, C. krusei, C. tropicalis, C. glabrata, C. dubliniensis and C. parapsilosis were isolated from MSU of DM patients in our study with random blood sugar ranging from 200 to $\geq 400 \mathrm{mg} /$ $\mathrm{dL}$. Isolation of non albicans Candida (NAC) from MSU of DM patients in our study corroborated the previous findings of Pandey and Pandey (2013) who reported the emergence of NAC in MSU of diabetic patients at Gwalior, India. The percentage occurrence of NAC (66.7\%) in MSU of DM patients was higher than C. albicans (33.3\%) and this agreed with the results of Ekpo et al. (2017) in Cameroon. Our observation also concurred with Kauffmann (2005) who reported that $>50 \%$ of urinary Candida isolates were NAC.

The susceptibility of Candida spp to antifungal agents is not often predictable, hence, investigating pathogenic Candida isolates against appropriate antifungal agents is required for effective treatments of patients. In our study, $20.5 \%$ Fluconazole and $20.5 \%$ Itraconazole resistant Candida spp were obtained and these values were $>13 \%$ Fluconazole and $18.5 \%$ Itraconazole resistant Candida spp reported by Sojakova et al. (2004). The resistance of Candida spp to Ketoconazole in this study correlated with the reports of Hugo and Russell (2007). Over-expression of CtERG11 or CDR1/CDR2 gene (Sanglard and Odds, 2002), over-expression of efflux proteins which act by pumping the drug out of the cell at a rate faster rate than how the drug enters the cell (Akinjogunla and Eghafona, 2012) and modifications of target enzymes have been attributed to the resistance of Candida spp to azole group of antifungal agents (Silva et al., 2011). A high percentage of Amphotericin B sensitive C. albicans, C. parapsilosis and C. tropicalis was 
observed and this substantiated the findings of Metin et al. (2011).

The transition of Candida isolate from a harmless commensal to potent pathogen is determined by several host predisposing factors and virulence attributes of organisms. The pathogenicity of Candida isolates is attributable to secretion of extracellular hydrolytic enzymes that act synergistically under favourable conditions (Silva et al., 2011). The occurrence of haemolysin, lipase and phospholipase producing Candida spp in this study corroborated the reports of Saha (2018) and Akinjogunla et al. (2019). Phospholipase facilitates Candida invasion by damaging host cellular contents and cleaves phospholipids of host cell membrane (Deorukhkar et al., 2014). Haemolysin aids in lysis of host erythrocytes and strips iron from haemoglobin molecules (Manns et al. 1994). Candida albicans and NAC exhibited biofilm formation and the occurrence of biofilm producing Candida spp agreed with Seneviratne et al. (2008). In our study, $14.3 \%$ coagulase producing C. tropicalis obtained was similar to $14.6 \%$ by Deorukhkar et al. (2014), but < $82.6 \%$ reported by Rodrigues et al. (2003). Coagulase binds plasma fibrinogen and activates a cascade of reactions that induce clotting of plasma (Rodrigues et al., 2003).

\section{CONCLUSION}

This study has shown the sociodemographic characteristics and risk factors in diabetic mellitus patients with or without candiduria; revealed the high sensitivity of Candida isolates to azole drugs and varied percentages of haemolysin, coagulase, biofilm, phospholipase and lipase producing Candida isolates in MSU samples and as well showed the necessity to continuously investigate pathogenic candida isolates against appropriate antifungal agents for effective treatments of asymptomatic candiduria in type 1 and 2 diabetes mellitus patients.

\section{ACKNOWLEDGEMENTS}

The authors vastly acknowledged all the participants and support staff who participated in this study.

\section{CONFLICT OF INTEREST}

The authors declares that there is no conflict of interest.

\section{AUTHORS' CONTRIBUTIONS}

Author AOJ designed the study, wrote the protocol and wrote the first draft of the manuscript. Authors DO and AYO wrote part of the manuscript and managed the analyses of the study. Authors EIU and EIJ managed the literature searches and performed the statistical analysis. All authors read and approved the final manuscript.

\section{FUNDING}

None.

\section{ETHICS STATEMENT}

The study protocol was in accordance with ethical procedures and recommendations of State Hospitals Management Boards guidelines.

\section{DATA AVAILABILITY}

The dataset used and/or analyzed during the current study are included in the manuscript.

\section{REFERENCES}

1. Abdulla MC, Jenner FP, Alungal J. Urinary tract infection in type 2 diabetic patients: risk factors and antimicrobial pattern. J Res Med Sci. 2015;3(10):25762579.

2. Abebe M, Adane T, Kefyalew K, et al. Variation of urine parameters among diabetic patients: A cross-sectional study. Ethiopians Journal of Health Sciences. 2019;29 (1): 877-882.

3. Akinjogunla OJ, Odeyemi AT, Olasehinde GI. Epidemiological studies of urinary tract infection (UTI) among post-menopausal women in Uyo Metropolis, South-South, Nigeria. Journal of American Science. 2010;6(12):1674-1681.

4. Akinjogunla OJ, Divine-Anthony O. Asymptomatic bacteriuria among apparently healthyundergraduate students in Uyo, South-South, Nigeria. Annual Review and Research in Biology. 2013;3(3):213-225.

5. Akinjogunla OJ, Eghafona NO. Mycological investigation in patients with acute otitis media. Scientific Journal of Microbiology. 2012;1(1):19-26.

6. Akinjogunla OJ, Divine-Anthony O, Akpan MM, Ogbonna FC. Virulence markers and antifungal susceptibility of vaginal yeast isolates from contraceptive and non-contraceptive users in Uyo, Nigeria. Journal of Experimental Research. 2019;7(1):33- 40.

7. Akinjogunla OJ, Divine-Anthony $O$, Nwadialor J. Candida isolates from high vaginal swabs of women of child bearing age: species distributions, 
virulence factors and antifungal susceptibility profile, Nigerian Journal of Scientific Research. 2017;16(4):5058.

8. Beers MH, Porter RS, Jones TV, Kaplan JL, Berkwits M. The Merck Manual of Diagnosis and Therapy. 18th edition. New Jersey: Merck Research Laboratories. 2006;1-85

9. Chakrabarti A, Ghosh A, Batra R, Kaushal A, Roy P, $\mathrm{H}$ Singh $\mathrm{H}$. Antifungal susceptibility pattern of nonalbicans Candida species and distribution of species isolated from candidaemia cases over 5-year period. Indian J Microbiol. 1996;104:171-176.

10. Clinical Laboratory Standards Institute. Method for antifungal disk diffusion Susceptibility testing of Yeasts; Approved Guideline-(2 ${ }^{\text {nd }}$ Ed). CLSI document M44-A2. Clinical Laboratory Standard Institute, Wayne: Pennsylvania. 2009.

11. Deorukhkar SC, Saini S, Mathew S. Virulence factors contributing to pathogenicity of Candida tropicalis and its antifungal susceptibility profile. International Journal of Microbiology. 2014;(ID 456878):1-6. https:// doi.org/10.1155/2014/456878

12. Donlan RM, Costerton JW. Biofilms: survival mechanisms of clinically relevant microorganisms. Clin Microbiol Rev. 2002;15:167-193. https://doi. org/10.1128/CMR.15.2.167-193.2002

13. Ekpo IT, Kechia FA, Iwewe YS, Ngueguim AD, Nangwat NC, Dzoyem JP. Species distribution and antifungal susceptibility profile of Candida spp isolated from urine of hospitalized patients in Dschang District Hospital, Cameroon. International Journal Biological and Chemical Sciences. 2017;11(3):1212-1221. https:// doi.org/10.4314/ijbcs.v11i3.23

14. Fernandes DJ, Jaidev MD, Dipthi NC. Utility of dipstick test (nitrite and leukocyte esterase) and microscopic analysis of urine when compared to culture in the diagnosis of urinary tract infection in children. International Journal of Contemporary Pediatrics. 2018;5(1):156-160. https://doi.org/10.18203/23493291.ijcp20175578

15. Francois LM, Duncan W, Bernhard H. Candida albicans pathogenicity mechanisms. Virulence. 2013;4(2):119128. https://doi.org/10.4161/viru.22913

16. Hassaneen AM, Ghonaim RA, Hassanin HM, Salama NA, Elgohary T. Different aspects of candiduria as an important nosocomial infection. Medical Journal of Cairo University. 2014;82(1):199-204.

17. Hu FB. Globalization of diabetes: The role of diet, lifestyle, and genes. Diabetes Care. 2011;34(6):12491257. https://doi.org/10.2337/dc11-0442

18. Hugo WB, Russell AD. Pharmaceutical Microbiology, (7th Ed). Blackwell Science. 2007;481.

19. Janifer J, Geethalakshmi S, Satyavani K, Viswanathan $V$. Prevalence of lower urinary tract infection in South Indian type 2 diabetic subjects. Indian Journal of Nephrology. 2009;19:107-111. https://doi. org/10.4103/0971-4065.57107

20. Kauffman CA. Candiduria. Clinical Infectious Diseases. 2005;41(Suppl 6):S371-376. https://doi. org $/ 10.1086 / 430918$

21. Lotfi-Kamran MH, Jafari AA, Falah-Tafti A, Tavakoli $\mathrm{E}$, Falahzadeh $\mathrm{MH}$. Candida colonization on the denture of diabetic and non-diabetic patients. Dental Research Journal. 2009;6(1):23-27.

22. Maahs D, West NA, Lawrence JM, Mayer-Davis EJ. Epidemiology of Type 1 Diabetes. Endocrinology and Metabolism Clinics of North America. 2010;39(3):481497. https://doi.org/10.1016/j.ecl.2010.05.011

23. Manns JM, Mosser DM, Buckley HR. Production of a haemolytic factor by Candida albicans. Infect Immun. 1994;62:5154-5156. https://doi.org/10.1128/ IAI.62.11.5154-5156.1994

24. Matulewicz RS, Meeks JJ. Blood in the urine (Hematuria). JAMA. 2016;316(14):1-2. https://doi. org/10.1001/jama.2016.4716

25. Metin DY, Hilmioglu-Polat S, Samlioglu P, DoganayOflazoglu B, Inci R, Tumbay E. Evaluation of antifungal susceptibility testing with microdilution and Etest methods of Candida blood isolates. Mycopathologia. 2011;172(3):187-199. https://doi.org/10.1007/ s11046-011-9413-y

26. Mukhtar Y, Galalain AM, Yunusa UM. A modern overview on diabetes mellitus: a chronic endocrine disorder. European Journal of Biology. 2019; 4(1):1-14.

27. Muller V, Viemann D, Schmidt M, et al. Candida albicans triggers activation of distinct signaling pathways to establish a proinflammatory gene expression program in primary human endothelial cells. Journal of Immunology. 2007;179:8435-8445. https://doi. org/10.4049/jimmunol.179.12.8435

28. Pandey M, Pandey A. Emergence of Non-albicans Candida in urine of diabetic patients at Gwalior (M.P.), India. IOSR Journal of Dental and Medical Sciences. 2013;4(5):11-14. https://doi.org/10.9790/08530451114

29. Rodrigues AG, Pina-Vaz C, Costa-de-Oliveira S, Tavares C. Expression of plasma coagulase among pathogenic Candida Species. J Clin Microbiol. 2003;41(12):57925793. https://doi.org/10.1128/JCM.41.12.57925793.2003

30. Saha S. Species distribution, virulence factors and antifungal susceptibility profile of Candida isolates from various clinical samples. Indian Journal of Basic and Applied Medical Research. 2018;7(3):206-212.

31. Sanglard D, Odds FC. Resistance of Candida species to antifungal agents: molecular mechanisms and clinical consequences. Lancet Infect Dis. 2002;2:73-85. https://doi.org/10.1016/S1473-3099(02)00181-0

32. Seneviratne $\mathrm{CJ}$, Jin $L$, Samaranayake $Y H$, Samaranayake LP. Cell density and cell aging as factors modulating antifungal resistance of Candida albicans biofilms. Antimicrob Agents Chemother. 2008;52:3259-3266. https://doi.org/10.1128/AAC.00541-08

33. Silva S, Negri M, Henriques M, Oliveira R, David WW, Azeredo J. Adherence and biofilm formation of nonCandida albicans species. Trends in Microbiology. 2011;19(5):241-247. https://doi.org/10.1016/j. tim.2011.02.003

34. Sobel JD, Fisher JF, Kauffman CA, Newman CA. Candida urinary tract infections: epidemiology. Clin Infect Dis. 2011;52(6):S433-S436. https://doi.org/10.1093/cid/ cir109

35. Sojakova M, Liptajova D, Borovsky M, Subik J. Fluconazole and itraconazole susceptibility of vaginal 
yeast isolates from Slovakia. Mycopathologia. 2004;157:163-169. https://doi.org/10.1023/ B:MYCO.0000020594.35357.b0

36. Soll DR. High-frequency switching in Candida albicans. Clin Microbiol Rev. 1992;5:183-203. https://doi. org/10.1128/CMR.5.2.183

37. Talaro KP, Talaro A. Foundations in Microbiology. (4th edn). McGraw-Hill companies, Inc, New York. 2002;899.

38. Yashavanth R, Shiju M, Bhaskar U, Ronald R,
Anita K. Candiduria: prevalence and trends in antifungal susceptibility in a tertiary care hospital of Mangalore. Journal of Clinical Diagnostics Research. 2013;7(11):2459-2461.

39. Zahra N, Rehman K, Aqeel R, Parveen A, Aka MSM. Assessment of urinary tract infection and their resistance to antibiotics in diabetic and non-diabetic patients. Bangabandhu Sheikh Mujib Medical University Journal. 2016;9:151-155. https://doi. org/10.3329/bsmmuj.v9i3.29511 